\title{
«Da poeta a poeta». Fortini lettore di Bassani tra critica e poesia.
}

"Da poeta a poeta ». Fortini lecteur de Bassani entre critique et poésie

Fortini's Reading of Bassani: Between Criticism and Poetry

\section{Gaia Litrico}

\section{(2)enEdition}

\section{Journals}

Edizione digitale

URL: http://journals.openedition.org/cei/3896

DOI: 10.4000/cei.3896

ISSN: 2260-779X

Editore

UGA Éditions/Université Grenoble Alpes

Edizione cartacea

ISBN: 978-2-37747-040-2

ISSN: $1770-9571$

\section{Notizia bibliografica digitale}

Gaia Litrico, ««Da poeta a poeta». Fortini lettore di Bassani tra critica e poesia.», Cahiers d'études italiennes [Online], 26 | 2018, online dal 28 février 2018, consultato il 26 mars 2021. URL: http:// journals.openedition.org/cei/3896 ; DOI: https://doi.org/10.4000/cei.3896

Questo documento è stato generato automaticamente il 26 mars 2021

(c) ELLUG 


\title{
«Da poeta a poeta» ${ }^{1}$. Fortini lettore di Bassani tra critica e poesia.
}

\author{
"Da poeta a poeta ». Fortini lecteur de Bassani entre critique et poésie \\ Fortini's Reading of Bassani: Between Criticism and Poetry
}

Gaia Litrico

\section{Premessa}

1 È noto in che modo e in che misura il lavoro di Franco Fortini sia attraversato dalla critica delle opere dei poeti e degli scrittori del suo tempo. Sulle pagine delle riviste e dei periodici che nel corso degli anni hanno ospitato le sue rubriche, egli ha ininterrottamente dialogato con i nomi più interessanti della letteratura italiana, non badando a mode e tendenze. Ricordiamo in particolare le «Rassegne» e le «Bibliografie» da lui curate per la rivista «Comunità», la quale nei primi anni del suo lavoro ha dato visibilità a tanta parte della sua riflessione. Ciò è documentato, oltre che dagli stessi scritti fortiniani, anche dalle testimonianze degli autori da lui recensiti, che hanno lasciato tracce del loro dialogo critico con Fortini nelle loro poesie e nella loro saggistica. Questo schema - che vede un autore riflettere sul proprio critico, producendo una sorta di 'critica del critico' - è piuttosto inconsueto e certifica la straordinarietà della critica letteraria di Franco Fortini. A questo proposito, tra i vari esempi che si possono riportare, le parole scritte da Mario Luzi sembrano essere le più incisive:

Egli fu e resta per me e penso per molti altri l'interlocutore ideale, cioè quello che si desidera intimamente avere perché non s'inganna sui tuoi intendimenti e ha tanta intuizione da farti luce su quanto di essi ti fosse rimasto in ombra e tanta competenza da conoscere a fondo i tuoi procedimenti e da coglierne la convenienza o l'improprietà dall'interno della situazione tua propria di cui fissa immediatamente i contorni ${ }^{2}$.

2 Ma a differenza di Mario Luzi, che nella dichiarazione del 1980 qui riportata esalta l'attività critica di Franco Fortini riconoscendogli la lucidità del lettore immune dagli 
inganni dell'artificio letterario, e a differenza anche di Vittorio Sereni, che nei versi di Un posto di vacanza riconosce al saggista fiorentino il ruolo positivo dell'«interlocutoreantagonista» che incoraggia il progredire della ricerca creativa dello scrittore ${ }^{3}$, Giorgio Bassani non ha consegnato al pubblico alcun parere sull'esercizio saggistico fortiniano. Tra le pagine dell'opera bassaniana sinora pubblicata, non è pervenuto né un giudizio sull'influenza che le parole dell'amico ebbero sulla sua produzione letteraria, né un commento più generale sul suo lavoro di critico. Il commento di Bassani alle critiche contenute nel corpus di otto articoli - recensioni e note di letture - che Fortini ha dedicato tra il 1950 e il 1962 sia alla sua prosa sia alla sua poesia, è da cercare piuttosto nel carteggio intrattenuto dai due scrittori nel corso degli anni ' 50.

In questa sede, dal momento che i saggi di Fortini sulla prosa ${ }^{4}$ di Bassani sono già stati studiati, è parso più utile ricostruire in un primo tempo, attraverso il carteggio, il pensiero di Bassani sulla critica fortiniana del primo periodo, dedicata esclusivamente alla sua poesia. In un secondo tempo, ci si concentrerà sulle motivazioni delle critiche mosse da Fortini a Bassani. Infine, si tenterà di verificare come, al di là di quelle critiche, Fortini compia un'altra, più dissimulata, opera di ricezione sul terreno della produzione lirica. Si individueranno perciò, tra i testi poetici degli anni ' 50 , dei punti di contatto tra i nostri due autori, da considerare spie rivelatrici dell'accoglienza della lezione bassaniana nel lavoro poetico di Fortini.

\section{Critiche e repliche sulla poesia di Bassani (1950-1952)}

Fortini ha portato avanti la sua riflessione critica su Bassani dal 1950 al 1962, concentrandosi in egual misura sui due generi praticati da Bassani in questo arco di tempo, la poesia e la prosa, e operando in due modi diversi. La maggior parte delle volte Fortini scrive commenti alle opere bassaniane che anno dopo anno sono date alla stampa: le più importanti sono quelle alla raccolta Un'altra libertà $(1952)^{5}$, al volume di racconti La passeggiata prima di cena $(1953)^{6}$, agli Ultimi anni di Clelia Trotti(1955) ${ }^{7}$. E ovviamente, la più celebre, nonché l'ultima, scritta all'indomani dell'uscita del capolavoro bassaniano, Il giardino dei Finzi-Contini ${ }^{8}$.

5 Altre volte invece Fortini inserisce "accenni telegrafici» ${ }^{9}$ all'opera bassaniana in analisi di più ampio respiro sulla letteratura di quel momento, mettendo in rilievo le caratteristiche peculiari dell'autore ferrarese rispetto alle altre voci letterarie coeve.

Proprio a questa seconda categoria appartengono due testi di Fortini sulla poesia di Bassani reperiti solo di recente e risalenti al $1950^{10}$, importanti perché svelano il decisivo ruolo della poesia in questo sodalizio e in questo dibattito critico.

7 Fino ad oggi, degli otto articoli di cui si accennava all'inizio, soltanto sei erano noti, di cui due soli dedicati alla poesia, il primo del 1952 (menzionato poco sopra) e il secondo del $1960^{11}$. Ma il recente lavoro di riordino e catalogazione del Fondo epistolare Bassani, ha portato, attraverso lo spoglio di lettere ricevute dallo scrittore ferrarese, all'identificazione di questi due nuovi testi che, oltre a permettere di antedatare di un paio d'anni l'inizio del lavoro del critico fiorentino su Bassani, rivelano come l'esordio di questo rapporto critico sia avvenuto sotto il segno della poesia ${ }^{12}$.

Secondo una catalogazione cronologica, il corpus critico sullo scrittore ferrarese può essere diviso in tre fasi. Il primo tempo è costituito dagli scritti fortiniani che si occupano delle prime prove poetiche. Il secondo è quello della prosa, in cui il lavoro 
critico si concentra sui racconti che compongono le Storie ferraresi. Il terzo è quello in cui viene riesaminato in un orizzonte più ampio il lavoro portato avanti nei due diversi generi letterari da Bassani. Fortini riepiloga qui dal suo punto di vista molte questioni sollevate dall'opera dell'amico: con uno scritto sulla poesia contemporanea uscito sul «Menabò» nel 1960, e con la recensione del Giardino nel 1962.

9 Si tratta di tre tappe di una parabola saggistica che vede un progressivo aumento delle critiche fortiniane, che evolvono verso un giudizio negativo, fino a giungere alla stroncatura contenuta nel già citato testo sui Finzi Contini o a quella contenuta nel testo sul Gattopardo ${ }^{13}$, che coinvolge oltre all'autore del romanzo, Giuseppe Tomasi di Lampedusa, anche Bassani, che come è noto svolse il ruolo di editor e curò la prefazione ${ }^{14}$. Di questa parabola si proporrà di prendere in esame solo un segmento, quello iniziale.

10 Il primo tempo occupa il periodo 1950-1952, biennio durante il quale Fortini si sofferma esclusivamente, con tre $\operatorname{articoli~}^{15}$, sulla produzione in versi dello scrittore ferrarese. Bassani si era presentato al pubblico nelle vesti di prosatore, nel 1940, con Una città di pianura ${ }^{16}$, ma non pubblicherà altri volumi di racconti $^{17}$ fino al 1953, anno della Passeggiata prima di cena ${ }^{18}$. Nel periodo postbellico, tra il 1945 e il 1952, lo scrittore ferrarese si dedica soprattutto a raccogliere in volume i versi della sua produzione lirica, facendosi conoscere dal pubblico e dai critici principalmente come poeta, attraverso una triade: Storie dei poveri amanti (I edizione del 1945 e II edizione accresciuta del 1946), Te lucis ante e Un'altra libertà ${ }^{19}$.

11 Fortini si occuperà come critico dei lavori poetici contenuti nell'ultima di queste raccolte, che comprende al suo interno anche Telucis ante $e^{20}$. Leggendo le lettere scambiate tra i due scrittori è palpabile sin da subito un senso di disorientamento in Bassani, che non si sente capito nelle sue ragioni poetiche dall'amico.

12 Nelle missive di questo periodo si individuano infatti toni di contrasto, di cui si ha un primo riscontro nella lettera scritta da Fortini a Bassani il 4 gennaio 1951: «Saprai la fine di Comunità. Spero tu non ne sia lieto perché ospitasse certi accenni telegrafici del Fortini. Soavi parla di rifarla».

13 L'allusione di Fortini a «certi accenni telegrafici» si riferisce a un articolo uscito pochi mesi prima, nell'ottobre del 1950, su "Comunità», dedicato al panorama poetico italiano contemporaneo, e in cui figurava anche il nome di Bassani, accanto a quelli di Bertolucci, Gatto, Penna, Parronchi e Arcangeli. Bassani in questo articolo vedeva definiti i suoi versi come «una musica artefatta e graziosa ${ }^{21}$ e, sebbene non siano giunte fino ad oggi lettere che lo testimonino, ci sono gli estremi per ipotizzare uno stupore sconcertato da parte sua. Quell'ironico «spero tu non ne sia lieto» lanciato da Fortini, induce infatti a pensare che Bassani avesse dimostrato le sue remore nei confronti di una definizione così limitante della sua poesia.

14 Come dichiara lui stesso in una nota al termine dell'articolo ${ }^{22}$, Fortini aveva letto il V Quaderno di «Botteghe oscure», in cui erano pubblicate alcune poesie di Bassani: L'effimera creatura, Bei colori, L'alba ai vetri, Ho visto in sogno ${ }^{23}$, che costituiscono per Bassani una tappa molto importante della sua parabola di poeta. Sono proprio queste, ad eccezione di Hovisto in sogno, le poesie usate dall'autore per argomentare l'evoluzione del suo percorso di poeta attraverso le prime tre raccolte poetiche nello scritto autoesegetico Poscritto, inserito nella raccolta L'alba ai vetri come postfazione, ma composto già nel $1952^{24}$. Queste poesie sono citate proprio per dimostrare un approdo a una seconda fase della propria produzione poetica: dopo aver pubblicato la prima 
raccolta Storie di poveri amanti, lavoro nato dalla grande sintonia con l'ambiente bolognese di Arcangeli, Rinaldi e Bertolucci ${ }^{25}$, Bassani, oramai giunto a Roma, desidera prendere una direzione differente, quella che guiderà la realizzazione delle successive raccolte, Te lucis ante e Un'altra libertà:

[...] il mio lavoro si svolse in direzione sostanzialmente opposta a quella del primo libro. C'era stata di mezzo la guerra - pensavo -, la guerra e la prigione. Se nei versi che stavo scrivendo volevo accogliere la nuova realtà che si imponeva al mio spirito, tutta la nuova realtà di me stesso e del mondo, allora dovevo lottare senza pietà, senza la minima condiscendenza nei confronti della mia natura, contro il ritagliato paradiso degli affetti primordiali da campirsi, inevitabilmente!, su uno sfondo d'idillio ${ }^{26}$.

Francesca Bartolini parla a questo proposito di «una decisa variatio di ispirazione in direzione di una più sofferta lettura del reale ${ }^{27}$, che però, stando a quanto si legge nell'accenno apparso sulla rivista olivettiana, il Fortini critico non riuscirebbe a cogliere.

Bassani sta ripensando il suo progetto poetico, sta recidendo il vincolo con una poesia legata alla linea bolognese, e quindi non solo il peculiare rapporto di fusione con «un paesaggio dai forti accenti regionali, quello padano, caratterizzato da "brughiere/ desolate", paludi nebbiose [...]», ma anche con «una posa squisitamente letteraria di ascendenza romantica $»^{28} \mathrm{e}$ infine con le «tracce decadenti» presenti in alcuni testi della prima raccolta ${ }^{29}$. Nel Poscritto Bassani desidera mostrarsi come un poeta dal profilo assai mutato, che ha maturato una coscienza «storica» ${ }^{30}$ prima assente: più responsabile nei confronti del suo tempo, egli liquida la «dolce squilla serale» ${ }^{31}$ per accogliere i segni della storia, «fulminei messaggeri» ${ }^{32}$ che lo aggrediscono e lo inducono a parlare dell'esperienza del carcere (Dal carcere), come delle visioni originate dall'immaginario bellico ancora vivido nella sua mente di poeta (Villa Glori). Fortini rimane invece almeno in parte ancorato all'atmosfera delle prove liriche precedenti allorché definisce Bassani un poeta che resiste sì alla moda «delle effusioni morbide $\mathrm{e}$ preraffaellite» ma solo «con l'ironia di una consumata retorica»"

17 Se Fortini non si accorge del cambiamento in «direzione opposta»" avvenuto tra la pubblicazione della prima raccolta e le due successive, la comunità letteraria invece sembra accorgersene: la raccolta Te lucis ante riceverà una segnalazione positiva in occasione di un premio letterario dell'ambiente capitolino, frutto degli investimenti del dopoguerra: il Premio Roma. Ma anche in quest'occasione lo sguardo critico fortiniano sembra essersi mostrato poco amichevole nei confronti di Bassani, o almeno così lo percepisce Bassani, come attestano gli scambi epistolari con Attilio Bertolucci ${ }^{35}$.

In una missiva del novembre 1950, che Bassani indirizza all'amico e confidente di lunga data, si leggono infatti parole di abbattimento: «Mi dispiace di Fortini, ma non ho mica voglia di chiedergli il ritaglio. Se tu mi sapessi dire il numero del giornale, sarebbe la cosa migliore. A patto però che non ti secchi troppo» ${ }^{36}$. Bertolucci il 7 novembre gli aveva scritto: «Volevo dirti d'un accenno di Fortini sull'«Avanti» a te premiato a Roma, come d'un esempio di premiato non tenero con i comunisti: un accenno che non so se amichevole o no o così così» ${ }^{37}$.

19 Sulla terza pagina dell'«Avantil», quotidiano dove Fortini teneva una rubrica di attualità e cultura, intitolata Schedario, era uscito il 2 novembre un articolo che rispondeva a una polemica sollevata dall'onorevole Mattei, secondo il quale i premi letterari, come anche le mostre e i concorsi, erano completamente monopolizzati dagli artisti di orientamento politico comunista che, proprio perché vincitori degli agoni artistici, 
godevano di una maggiore diffusione e promozione, finendo per «avvelenare la mente del pubblico di massa» ${ }^{38}$. Fortini, desiderando dimostrare la fallacità di questa tesi, portava come esempio il concorso del Premio Roma, tenutosi solo qualche mese prima, dove Ungaretti aveva ottenuto il primo posto con La terra promessa e Bassani, che era riuscito a partecipare con la raccolta Te lucis ante, una segnalazione per merito ${ }^{39}$. Fortini, nel rispondere sulle pagine dell' «Avantil», aveva sottolineato come né Ungaretti né Bassani potessero essere classificati come artisti comunisti e aveva ironizzato su quanto poco di sovversivo vi fosse nelle loro opere.

Probabilmente Fortini, nel definire la poesia di Bassani 'non sovversiva', non intendeva in alcun modo colpire il poeta, che del resto non ambiva a ricevere una qualifica del genere, eppure finiva per battere su un tasto dolente: il riconoscimento dell'impegno politico presente nella sua poesia. Proprio nel momento in cui Bassani tenta la transizione verso una poesia engagée, in grado di rappresentare la condizione dell'uomo all'indomani della guerra e in cui tenta di dar voce all'esperienza delle lotte per la liberazione dall'occupazione nazista, il gesto del critico fiorentino diventa simbolicamente una delegittimazione della presenza del tema politico nella poesia di Bassani, proprio agli occhi di un pubblico che il poeta potrebbe desiderare raggiungere. $\mathrm{E}$ se in tale occasione Bassani non mostra la sua delusione al diretto interessato, questa riaffiora in una lettera a Fortini del marzo 1952, in cui è fatto riferimento ai «compagni socialisti», che lo scrittore teme «si facciano del libro [...] un'immagine poco simpatica e poco probabile» ${ }^{40}$.

21 In questa lettera Bassani allude a un passo di una prima versione della recensione di Fortini alla sua raccolta poetica Un'altra libertà. Anche se Bassani lo aveva preparato con una lettera in cui affidava alle cure esegetiche dell'amico con una certa sollecitazione il suo libro («Hai letto il mio libro? Ti è piaciuto? Tu sei dei pochi che lo possono capire: te lo raccomando») $)^{41}$, ancora una volta, come già nei precedenti brevissimi interventi, la sua nuova poetica non è capita. Fortini omette di indicare al lettore che Bassani è stato in carcere e sottolinea della raccolta solo gli elementi caratteristici di una letteratura del disimpegno, iperletteraria e dalla forte carica solipsistica:

È questa una poesia sulla poesia, una poesia di ironia malinconica, la dolce malia del prim'atto della Bohème riscattata da un sorriso amaro e cosciente; essa si sostiene per la sua discrezione, per quella sua emozione sorridente che accetta quindi le convenzioni preziose e l'equivoca moda di chi allude (come certi arredamenti o toilettes) al 1905-1912 ${ }^{42}$.

Paola Italia già si è soffermata sul senso di ingiustizia provato da Bassani nel non vedere riconosciuto il suo passato di antagonista del regime, di prigioniero politico ( $« \mathrm{Se}$, data la sede [...], ti pareva necessario dare qualche notizia biografica, potevi pur dire che sono stato in galera, a suo tempo; tanto più che la galera torna, nel libro, come ossessione e memoria fondamentale» $)^{43}$, che porterà a una rielaborazione della recensione $\mathrm{e}$ a un conseguente cambio di destinazione editoriale (non verrà più pubblicata sulle pagine del quotidiano socialista, bensì su «Comunità»). Il confronto della bozza della prima recensione, rinvenuta nell'Archivio epistolare Bassani, con la versione definitiva, mostra infatti discreti miglioramenti, sia nel tono, che si fa più analitico e meno frivolo (ad esempio, l'incipit della bozza presentava Bassani attraverso la partecipazione al mondo del cinema in un film del 1952, elemento che allo scrittore ferrarese era sembrato fuori luogo), sia in alcune argomentazioni critiche che si dilungano con maggiore puntualità sui versi (ad esempio rintracciando attraverso le fonti la presenza della tradizione $)^{44}$. Fortini cerca insomma di giustificare in modo più 
analitico le sue posizioni, sfumando qualche punto sgradito a Bassani e aggiungendo elementi obbligatori, come l'esperienza del carcere, ma non cambiando in modo sostanziale la sua opinione.

La fine del primo tempo della critica fortiniana è rappresentata bene in una frase scritta da Bassani per lettera all'amico, in cui si riassume il suo sentimento: «Mi sentii [...] frainteso, e con poca giustizia, proprio da te, che eri dei rari ai quali speravo di poter comunicare» ${ }^{45}$.

Bassani è insoddisfatto dell'accoglienza critica dell'amico, ma forse proprio queste ostilità gli consentono (o lo costringono) a conquistare durante quel biennio una maggiore chiarezza sul suo progetto poetico.

Se in occasione dei primi interventi del 1950, Bassani, nonostante il dispiacere, mostra delusione e scontento senza dilungarsi in termini teorici sulla questione, nel marzo 1952 si assiste infatti a un cambiamento: Bassani sembra aver maturato una maggiore consapevolezza sulla sua poesia, probabilmente anche perché sollecitato dal pungolo fortiniano, e sostiene con convinzione le sue posizioni, suggerendo quelle prospettive ermeneutiche che a Fortini erano sfuggite. In primo luogo ciò è testimoniato dal carteggio che vede un aumento della quantità delle missive scambiate (se di norma il ritmo dello scambio epistolare all'interno del carteggio Bassani-Fortini è di una lettera al mese, dal 20 al 27 marzo subisce un notevole aumento, registrando tre missive in una sola settimana), caratterizzate da una dialettica fertile e produttiva.

In secondo luogo, il cambiamento è testimoniato dal contenuto di Poscritto, il testo a cui si accennava sopra, steso proprio nello stesso anno della polemica sulla recensione a Un'altra libertà, il 1952. In questo testo Bassani illustra le ragioni del suo poetare, dimostrando di aver rafforzato, anche per via del dibattito epistolare con l'amico, la coscienza dei propri esiti artistici.

27 Sembra lecito allora ipotizzare che proprio il confronto con Fortini abbia ispirato Poscritto e abbia convinto il poeta ferrarese a rilasciare alcune dichiarazioni programmatiche sulla sua poesia, a parlare di sé come scrittore, a rivendicare un determinato carattere per i suoi versi.

28 I punti esposti da Bassani in questo testo ci riconducono infatti ai due fronti della querelle con Fortini: da una parte le poesie citate da Bassani coincidono con quelle con cui Fortini aveva illustrato le sue considerazioni nel 1950, e ci fanno dunque pensare a una replica a lui direttamente indirizzata; dall'altra l'insistere sullo scarto tra una poesia prebellica, di tipo iperletterario e di matrice decadente, e una postbellica, segnata dalla guerra e maggiormente impegnata, indica con precisione ciò che il poeta vorrebbe far cogliere da Fortini nella sua opera.

29 Si può dunque concludere che questo testo sia stato scritto appositamente per replicare a Fortini, che esso costituisca un chiarimento che Bassani destina indirettamente all'amico, nella speranza di indurlo a modificare il suo sguardo critico sulla sua poesia.

\section{Poetica di un autore, strategia di un critico}

30 Vi è sostanzialmente una ragione dietro all'attitudine critica che Fortini adotta rispetto al lavoro in versi di Bassani, ed è attinente al percorso poetico dello stesso Fortini; è legata ad alcuni limiti che Fortini scorge nelle prime prove della propria poesia d'anteguerra, delle quali ha intenzione di liberarsi attraverso un cambio di direzione 
che si concretizzerà in un primo momento con la raccolta Foglio di Via e che proseguirà ancora in forma di perpetua ricerca espressiva fino alla pubblicazione di Poesia ed errore, raccolta dopo la quale, secondo lo studioso Francesco Diaco, si inaugura una nuova fase $e^{46}$.

Questa ricerca di una propria voce poetica, nuova rispetto alla produzione d'anteguerra, avviene durante il decennio in cui Fortini dialoga con Bassani, che va dal 1949 al 1959, e il carteggio ne racconta l'evoluzione. Si tratta perciò di un dialogo proficuo per i due poeti, che in due sensi diversi fa maturare le loro rispettive consapevolezze letterarie.

Giovanni Raboni aveva individuato nel poeta fiorentino un'attitudine, particolarmente calzante per questo discorso:

[...] la tendenza a creare singoli rapporti 'di coppia', a stabilire con altri singoli poeti dei rapporti di comprensione conflittuale, di alterità complice, di particolare intensità e di straordinaria, illuminante rilevanza storico-culturale. Il rapporto Fortini-Sereni, il rapporto Fortini-Pasolini sono già molto noti [...]. Ma credo che molto importanti potranno rivelarsi, quando ci sarà stato modo di conoscerli $\mathrm{e}$ studiarli, anche altri rapporti, altre 'coppie', assai meno ufficiali ${ }^{47}$.

Quella Bassani-Fortini sembra essere proprio una delle coppie «meno ufficiali» di cui Raboni auspicava il recupero.

Le osservazioni che Fortini scrive in quanto critico sulla poesia contemporanea e, attraverso una "comprensione conflittuale» e un'«alterità complice», anche su Bassani, riflettono un'idea di poesia che Fortini ha messo a punto proprio negli anni precedenti a partire anche dalla sua stessa esperienza di poeta.

Il rapporto tra Bassani e Fortini, infatti, che si sviluppi sul piano critico o su quello editoriale (Bassani per tutto il decennio degli anni ' 50 sarà il punto di riferimento per le pubblicazioni dei suoi testi poetici), è sempre animato dal confronto su questioni poetiche che stanno dietro a vari episodi del loro dialogo.

Nel caso di Fortini la convivenza del poeta accanto al critico è uno schema consueto, già sperimentato negli anni fiorentini dell'esordio letterario. Lo testimonia il fatto che lo scrittore «non nasce saggista», come nota Lenzini ${ }^{48}$, ma sperimenta prima l'attività versificatoria, pubblicando su alcune riviste fiorentine le sue liriche ${ }^{49}$, e poi, poco dopo, inaugura quella del critico, il cui debutto avviene alla fine degli anni '30 attraverso prove dedicate anche all'analisi dei grandi poeti del periodo - basti ricordare a questo proposito i testi dedicati a Ungaretti e Quasimodo ${ }^{50}$.

36 Nel 1946, a distanza di qualche anno dalla loro composizione, le poesie scritte all'inizio degli anni ' 40 destano in Fortini disorientamento e un senso di rifiuto, perché avvertite come frutto della formazione giovanile, oramai inattuale e superato. Scritte prima della guerra rappresentano un'esperienza passata, scaturita da un contesto segnato da un «disimpegno iperletterario e decadente» ${ }^{51}$, la Firenze degli anni '30. Nota acutamente Luca Daino che c'è un elemento rivelatore della frattura creata da Fortini tra il presente del dopoguerra e la sua adolescenza e formazione nel capoluogo toscano: nella Prefazione all'edizione di Foglio di via del 1967, nel ripercorrere il suo esordio letterario, Franco Fortini, proprio per allontanarsi dal poeta giovane e inesperto usa la terza persona, passando all'adozione della prima persona solo con il sopraggiungere nel racconto del periodo della guerra ${ }^{52}$.

37 Anche per lui, come per Bassani, gli anni bellici segnerebbero una svolta, uno spartiacque, in seguito al quale si deve operare un cambiamento. Non solo le esperienze 
concrete della partecipazione alla Resistenza e alla Repubblica della Valdossola ma anche gli incontri intellettuali avvenuti durante questo periodo permettono a Fortini di emanciparsi dal suo passato e rinascere all'insegna di nuovi orizzonti intellettuali. «[...] il primo nonché fondamentale tentativo compiuto da Fortini in questa direzione è rappresentato dal suo libro d'esordio Foglio di via e altri versi», all'interno dei quali però si configurano due esperienze di differente segno ${ }^{53}$, quella della formazione fiorentina e quella dell'esilio e della lotta partigiana durante la guerra. Fortini, come spiega ancora Daino, provvede a liberarsi del suo passato poetico attraverso una precisa disposizione non cronologica dei pezzi all'interno della raccolta, ovvero inserendo le liriche anteriori all'avvento della guerra nella sezione Elegie, in posizione centrale, preceduta e seguita dalle altre due sezioni che ospitano invece i testi postbellici e resistenziali.

In questo modo la parte da ripudiare perde importanza e viene messa in secondo piano, delegittimata e depotenziata, rispetto ai brani che la precedono e la seguono ${ }^{54}$. Ma quali sono in particolare gli elementi di questa produzione poetica giovanile che Fortini vuole rinnegare, diminuendone l'importanza all'interno di Foglio di via? Rispondere a questo quesito aiuterà a spiegare le posizioni critiche fortiniane sulla poesia del dopoguerra dell'amico Bassani. La poesia riunita in Elegie è una poesia incentrata tutta sul dramma interiore vissuto dall'io lirico e in numerose occasioni si presenta come dialogo con un tu in absentia, dalle implicazioni sentimentali ${ }^{55}$. Come dice Daino, si tratta di una concezione egotistica ${ }^{56}$ dell'attività poetica; la voce lirica si declina attraversando la dimensione degli affetti, che sembra essere quasi totalizzante, e spesso la realtà interiore è in un rapporto idilliaco con la natura.

Questi sono sicuramente tratti comuni a diverse voci poetiche del Novecento, e lo stesso Bassani presenta alcune analogie con una concezione poetica di questo tipo. Facendo un breve sondaggio delle relazioni presenti tra le Elegie fortiniane e Storie dei poveri amanti, ad esempio, si riscontra una ricorrenza delle medesime immagini e occorrenze lessicali. L'amante, ad esempio, è resa con immagini rimandanti a elementi 'celesti' della natura (Fortini: «i cieli che schiude il tuo sguardo» ${ }^{57}$; Bassani: «tu respiri coi cieli $\left.\aleph^{58}\right)$; il paesaggio, con cui c'è una forte compenetrazione, è proposto da entrambi con i medesimi motivi tratti dal mondo vegetale (Fortini: «erba nuova»" ${ }^{59}$; Bassani «oscura erba» ${ }^{60}$, «calde erbe infinite $\left.»^{61}\right)$; e entrambi poi traducono in versi motivi come l'infanzia, il pianto, il sonno, per non citare che i più evocativi.

Ma se Bassani, nel 1952, in Poscritto, dichiarava di aver voltato pagina rispetto a Storie dei poveri amanti, il suo «rifiuto» non negava però «la possibilità di un recupero futuro» ${ }^{62}$, come nota Francesca Bartolini.

41 Nella prima sezione di Un'altra libertà, corrispondente eccetto alcune variazioni alla precedente raccolta del 1947 Te lucis ante, vengono infatti inseriti alcuni testi che F. Bartolini definisce vicini alla linea originaria ${ }^{63}$, quella della prima raccolta, brani portatori perciò di immagini campestri e temi che mostrano i diversi modi della sofferenza dell'io immerso in una natura onnipresente ${ }^{64}$. A Fortini proprio questi componimenti devono essere sembrati troppo vicini a quella produzione giovanile che lui aveva con tanta cura cercato di mettere al margine. «La seconda parte del libro (Dal profondo) ci par migliore della prima (Te lucis ante) ${ }^{65}$ precisa infatti Fortini nella bozza della recensione a Un'altra libertà che è stata rinvenuta tra le lettere di Bassani, confermando la difficoltà ad apprezzare quanto gli ricorda linee espressive dalle quali solo poco prima egli stesso si era emancipato. Al di là di questa sentenza, il resto della bozza e la stessa recensione definitiva scritta poco dopo dimostrano il desiderio di 
Fortini di liquidare quegli errori giovanili: «[...] l'assonnarsi nel crepuscolo, la dolente umiliazione, le luci attraverso il pianto, la straziante e dolce capitolazione, la discesa verso un sonno proustiano» esistono secondo Fortini solo in dipendenza di «una situazione emozionale che mai si dialettizza con un suo diverso e contrario» ${ }^{66}$; la poesia finisce così per essere costituita da mere sospensioni emotive «che rinunziano alla interrogazione interna» ${ }^{67}$. Il pathos dell'io, secondo Fortini, è il centro dell'opera che sembra rimanere intrappolata proprio in questa dimensione solipsistica e autoreferenziale. Si è accennato poco sopra come Fortini avesse sentito sulla propria pelle questa impasse e avesse deciso di uscirne attraverso l'instaurazione di un legame tra l'io e la dimensione politica e collettiva, che nelle sezioni Gli Anni e Altri versi di Foglio di Via è ravvisabile nelle tematiche resistenziali e civili che vi sono ritratte. Per quanto riguarda il modo in cui Fortini giunge a queste consapevolezze, meriterebbe di essere messo in luce il ruolo avuto dalle teorie marxiste conosciute durante l'esilio svizzero («Ho letto Lenin e Marx / non temo la rivoluzione») ${ }^{68}$. In effetti, se è vero che sia Bassani sia Fortini scelgono di voltare pagina dopo aver conosciuto la lotta al regime fascista e gli eventi della guerra, lo fanno però in modi diversi: mentre Fortini intraprende un percorso di rinnovamento sotto la guida dei testi sopra menzionati, Bassani sceglie una rottura meno radicale, in cui, pur essendo presente un distacco da certi elementi meramente autoreferenziali in favore di «tutta la nuova realtà» - che egli mette in primo piano «contro il ritagliato paradiso del gusto e della cultura, contro il facile paradiso degli affetti primordiali da campirsi, inevitabilmente!, su uno sfondo di idillio» ${ }^{69}$ - tuttavia intende diversamente l'idea di "verifica dell'individuo nella storia collettiva ${ }^{70}$. In altre parole, la dimensione privata nella sua produzione lirica non è messa in discussione quanto in quella di Fortini.

Insomma, la recensione di Fortini a Un'altra libertà e la sua strategia critica possono essere inscritte all'interno dell'operazione di rifiuto dell'esperienza letteraria prebellica, già messa in atto nella costruzione della raccolta Foglio di Via e che il poetacritico continua a mettere a punto nel corso di tutta la prima fase della sua poesia, passando dal piano poetico a quello critico.

\section{«Semmai distratto recensore, son tuttavia buon lettore ${ }^{71}$ : la ricezione nella poesia di Fortini}

43 Si è visto come attraverso la partecipazione all'esperienza della guerra Fortini abbia messo a punto alcuni concetti chiave della sua poetica, quello del perpetuo confronto del poeta con la Storia, ad esempio, e ne abbia rifiutati altri, come il solipsismo autoreferenziale e l'iperletterarietà con cui si era confrontato nell'età giovanile. Si è visto inoltre come tali idee sulla poesia, oltre che essere perseguite nella propria scrittura in versi, abbiano animato anche le pagine che recensiscono i poeti di quel periodo, tra cui Giorgio Bassani, al quale è rimproverato un racconto eccessivamente emozionale, non inserito in un contesto storico che trascenda l'io poetico, un racconto che offre, per dirla con Guido Mazzoni, «pochi frammenti di vita [...], riducendo il mondo esterno a paesaggio, separando la prima persona dai campi di forze psichiche e sociali che la attraversano» ${ }^{72}$.

La fine del dopoguerra e il conseguente cambiamento della realtà storica costringono però il poeta Fortini a modificare di nuovo la direzione del suo lavoro in versi. Pur mantenendo ferme le riflessioni poetiche conseguite in seguito all'esperienza bellica, 
chiusa la stagione della Resistenza e «venendo meno lo scenario storico del conflitto, con il suo portato d'immagini apocalittiche» ${ }^{73}$, il poeta, come scrive Diaco, «si ritrae come l'uomo di cultura solo e insonne che continua a scrivere [...]; dal momento che l'azione gli è preclusa deve accontentarsi di descrivere e riflettere» ${ }^{74}$. Pertanto nelle poesie degli anni' 50 in primo piano è di nuovo presente l'io: «è Il ritorno - così s'intitola la prima sezione di Al poco lume- [...], ad una dimensione privata ed esistenziale» ${ }^{75}$.

Nei versi scritti tra la fine degli anni '40 e i primi anni '50 si riflette perciò questa trasformazione del carattere poetico fortiniano, ovviamente non da intendersi come un ritorno all'esperienza giovanile - Fortini ha ormai superato definitivamente i versi delle Elegie - ma come un allentamento del serrato confronto con la Storia, al fine di concentrarsi e prendersi cura della sfera legata alla propria vita interiore. Per non confondere la poesia della sezione elegiaca di Foglio di via con la strategia poetica che regola gli anni successivi, a cavallo tra la fine dei' 40 e l'inizio dei '50, è utile la definizione che offre Diaco nel suo studio, per il quale il lavoro di questi anni sarebbe una «correzione esistenzialista allo storicismo» ${ }^{76}$. I versi si riempiono infatti di interrogativi di stampo etico, di riflessioni metaletterarie, di lunghi sguardi che contemplano quanto accade fuori e vi riflettono sopra ${ }^{77}$. Il poeta è da solo, non più inserito in una collettività, come era invece in gran parte di Foglio di via, e da questa posizione di isolamento nasce un canto di impronta più individuale.

Avviandoci a concludere, sembra allora lecito avanzare l'ipotesi che in questa fase il poeta fiorentino abbia potuto approdare a una nuova poetica proprio grazie alla ricezione critica di Bassani. In altre parole, Bassani sarebbe stato un utile termine di confronto per l'operazione poetica di stampo esistenzialista che Fortini desidera mettere in atto.

Il poeta fiorentino, del resto, confessa apertamente all'amico il suo desiderio di rinnovamento in una lettera del 1949: «[...] c'è tutto da rifare, la maggior parte dei miei versi non resiste a una vera severità, e la cosa che resta da fare è scriverne di migliori $[\ldots])^{78}$.

48 Fortini, chiedendo consigli («[...] Il "Falso sonetto" ha gli ultimi due versi che mi fanno impazzire. Vedi tu, anche qui, di usare quel che credi in sostituzione di quel che ti pare meglio») ${ }^{79}$ e giudizi («Caro Bassani, non invitato, ma su consiglio di Soavi, ti mando otto poesie. Ti prego di scrivermene qualcosa» $)^{80}$ sul suo lavoro, riesce in effetti nel suo intento e anima con nuove questioni la sua poesia: una di queste è «il ricordo dell'infanzia e [...] l'irruzione di un tempo 'altro', carico di futuro» ${ }^{81}$ che, come nota Lenzini, è presente nella poesia Per questa luce, composta nel 1950.

Tema che in quegli stessi mesi anche Bassani affronta proprio nella sezione della raccolta Un'altra libertà giudicata migliore da Fortini, ovvero Dal Profondo. Ad esempio la poesia L'alba ai vetri del 1950 lo sviluppa enfatizzando come l'arrivo del futuro comporti la fine di un'epoca passata e sia fonte di dolore per il poeta:

[...] Eri tu che passavi, vita, tu, vita mia, tu che sopravvenivi, innocente futuro.

«Empio evo venturo che premi dalle porte»

dicevo io, con lacrime più soavi che amare, «dimentica il mio nome $[. ..] »^{82}$. 
Nelle pagine di Dal Profondo più volte ricorre il desiderio di tornare all'«isola del passato»" ${ }^{83}$, che l'«avvenire» ${ }^{84}$ rischia di portare via, e quello di trattenere «i primi ricordi che la febbre degli anni» rischia di mettere a tacere ${ }^{85}$.

Questi versi sembrano condividere con alcune liriche di Fortini del 1950 una posizione comune sul rapporto con il futuro. In Per questa luce, appunto, si legge di uno stato di attesa del poeta che tremante aspetta, stringendo la mano di una persona a lui vicina.

Che il tema dell'infanzia e quello del sopraggiungere del futuro siano cari a Fortini si può notare anche dal fatto che ritornano nell'ultimo accenno critico sulla poesia bassaniana, pubblicato nel 1960 sulla rivista «Il Menabò». In questa sede il critico si sofferma proprio sul senso più profondo che alberga nella raccolta Un'altra libertà, su quel «tremore vero, un vero infantile pianto di adulto, in quegli angoli di giardino paradisiaci, in quel passare della vita tra i pifferi di un "empio evo venturo" ${ }^{86}$. Se si osserva con attenzione la costruzione del periodo, traspare un diretto coinvolgimento del Fortini poeta.

Infatti, se tutte quelle menzionate in questo passo sono immagini tratte da poesie bassaniane, il primo elemento dell'elenco, il «tremore vero», fa eccezione, non risultando essere una figura del repertorio dal poeta ferrarese ${ }^{87}$. È stato invece notato da Lenzini e Diaco come tale lemma sia caratteristico di alcune poesie fortiniane dell'inizio degli anni ' 50 relative proprio al senso di attesa e all'arrivo del futuro ${ }^{88}$.

Con l'atto simbolico di scegliere una parola proveniente dal proprio immaginario poetico per ritrarre la poesia di Bassani si conclude il capitolo della critica di Fortini sulla poesia dell'amico.

L'occorrenza citata sembra suggerire l'esistenza di una zona di contatto tra i due autori: il poeta Fortini all'inizio degli anni ' 50 aveva forse già capito che «il solo modo serio di dire "noi" è dire "io" ${ }^{89}$ e aveva individuato in Bassani uno dei compagni con cui concretizzare in versi quella momentanea immersione nella propria esperienza, prima

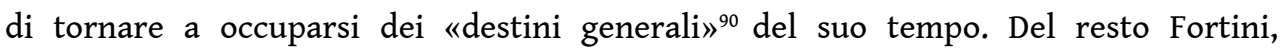
consapevole di non averlo dimostrato sul fronte critico, in una lettera aveva tenuto a precisare quanto prezioso considerasse il confronto poetico con l'amico: «Ti prego di credere alla mia stima; da poeta a poeta, se non son parole troppo grosse» ${ }^{91}$.

\section{NOTE}

1. La frase è tratta da una lettera che Fortini invia a Bassani il 25 marzo 1952. Attualmente è conservata presso l'Archivio dello scrittore fiorentino, donato nel 1994 dagli eredi al Centro Studi Franco Fortini e custodito presso la Biblioteca di Lettere dell'Università di Siena. Ringrazio il prof. Lenzini, direttore dell'Archivio del Centro Studi Fortini, e la dott.ssa Nencini per la disponibilità con cui mi hanno permesso di consultare, studiare e citare il materiale epistolare conservato a Siena. Inoltre sono riconoscente a Paola Bassani e al fratello Enrico per avermi concesso di studiare e riportare in questa sede alcune parti della corrispondenza del padre. Ringrazio anche Paolo Lagazzi che mi ha gentilmente concesso di citare la corrispondenza di 
Attilio Bertolucci con lo scrittore ferrarese, permettendo di sciogliere alcui nodi attorno alla storia dei testi critici di Franco Fortini sull'opera di Bassani.

2. M. Luzi, De amicitia (quadam), in C. Fini (a cura di), Per Franco Fortini. Contributi e testimonianze sulla sua poesia nel sessantesimo dell'autore, Padova, Liviana Editrice, 1980, p. 116.

3. «Calvino, Pasolini, Sereni, in Fortini avvertirono non il lettore-fiancheggiatore o un pur acutissimo interprete, ma l'interlocutore-antagonista di cui avevano bisogno per far avanzare la propria ricerca creativa» (L. Lenzini, Introduzione a Fortini saggista, in Id., Un'antica promessa, Macerata, Quodlibet, 2013, p. 43). Al rapporto tra Franco Fortini e Vittorio Sereni Luca Lenzini ha dedicato anche un altro contributo, Due destini. Sul carteggio Sereni-Fortini, in R. Esposito (a cura di), Vittorio Sereni, un altro compleanno, Milano, Ledizioni, 2014, pp. 299-315.

4. Nel 2011 Paola Italia, in uno studio sui materiali dal carteggio Bassani-Fortini che erano accessibili a quell'altezza, offriva anche una panoramica sulle recensioni fortiniane a Bassani, in particolare quelle dedicate a Una lapide in via Mazzini, Gli ultimi anni di Clelia Trotti e Il giardino dei Finzi Contini. Si rinvia a P. Italia, Tra poesia e prosa: un percorso dal carteggio Bassani-Fortini (1949-1970), in A. Perli (a cura di), Giorgio Bassani: la poesia del romanzo, il romanzo del poeta, Ravenna, Giorgio Pozzi Editore, 2011, pp. 57-80. Inoltre, nel 2015 Raffaele Manica ha preso in esame la recensione fortiniana al Giardino all'interno del suo lavoro dedicato alla riscrittura che Bassani ha compiuto del romanzo di Micòl (il testo è consultabile a questo link <https://f.hypotheses.org/ wp-content/blogs.dir/326/files/2016/01/Manica.pdf>).

5. F. Fortini, Poesia di Bassani, in Bibliografia letteraria, «Comunità», a. VI, $\mathrm{n}^{\circ} 74$, giugno 1952, pp. 73-77.

6. F. Fortini, Bassani e D'Arzo, in Narrativa dell'annata, in Bibliografia letteraria, a cura di F. Fortini, «Comunità», a. VII, nº 20, settembre 1953, p. 46.

7. F. Fortini., Lettera. I racconti di Bassani, «Lo Spettatore Italiano», a. VIII, 7 luglio 1955, pp. 296-298.

8. F. Fortini, «Dal nulla tutti i fiori: il romanzo di Bassani», «Comunità», a. XVI, marzo-aprile 1962, pp. 42-46, poi in Saggi italiani, Bari, De Donato, 1974, ora in Saggi ed epigrammi, cit., pp. 709-719.

9. L'espressione è tratta da una lettera inviata da Fortini a Bassani il 4 gennaio 1951. Attualmente è conservata presso l'Archivio Eredi Bassani, sito a Parigi. Già studiato da Paola Italia nel 2011 (Tra poesia e prosa: un percorso dal carteggio Bassani-Fortini (1949-1970), cit., pp. 67-80) e da L. Daino (Come nasce un libro di poesia? Bassani e Sereni editori e poeti, in I. Crotti, E. Del Tedesco, R. Ricorda e A. Zava (a cura di), Autori, lettori e mercato della modernità letteraria, Pisa, ETS, 2011, pp. 211-220), è di prossima pubblicazione l'edizione integrale e commentata del carteggio, a cura di chi scrive.

10. F. Fortini, Su questo momento di poesia, in Bibliografia letteraria, a cura di F. Fortini, "Comunità», a. IV, no 9, settembre-ottobre 1950, pp.63-67; F. Fortini, Schedario, «Avanti!», 2 novembre 1950, p. 3 .

11. F. Fortini, Le poesie italiane di questi anni, «Il Menabò», $\mathrm{n}^{\circ}$ 2, 1960, pp. 103-142. Il testo sarà poi ripreso in Saggi italiani, Bari, De Donato, 1974.

12. Oltre ai documenti utili per uno studio della ricezione di Bassani da parte degli intellettuali del suo tempo, come Fortini, anche il carteggio Bassani-Fortini è stato arricchito da nuova documentazione, cinquanta nuove lettere, rinvenute grazie al recente lavoro di riordino del Fondo Epistolare Bassani condotto dalla sottoscritta, da Lucia Bachelet, Silvia Datteroni, Costanzo Cafaro, Brigitta Loconte. Vorrei ringraziare Paola Bassani e il fratello Enrico, che si occupano del patrimonio documentario del padre, assicurandone una sempre maggiore divulgazione, e la Prof.ssa Paola Italia che ha diretto i lavori archivistici, permettendo di avanzare così nelle ricerche su questo autore.

13. F. Fortini, Contro il "Gattopardo», in Id., Saggi ed epigrammi, a cura di L. Lenzini, Milano, Mondadori, 2003, pp.720-730. 
14. G. Bassani, Prefazione, in G. Tomasi di Lampedusa, Il Gattopardo, Milano, Feltrinelli, 1958, pp. 7-13, poi in G. Bassani, Opere, a cura di R. Cotroneo e P. Italia, Milano, Mondadori, 2009, pp. 1156-1161.

15. F. Fortini, Su questo momento di poesia, cit., pp.63-67; F. Fortini, Schedario, cit., p. 3; F. Fortini, Poesia di Bassani, cit., pp. 73-77.

16. G. Marchi, Una città di pianura, Milano, Officina d'Arte Grafica A. Lucini \& Co, 1940. All'interno del volume prevale la prosa, ma Bassani vi inserisce anche una poesia, Ancora dei poveri amanti, pp. 133-135.

17. Questo non vuol dire che la prosa scompaia dall'officina bassaniana. Sebbene non escano in volume, in realtà alcune prose narrative dello scrittore ferrarese appaiono sui periodici e sui quotidiani del dopoguerra (cfr. nota seguente).

18. G. Bassani, La passeggiata prima di cena, Firenze, Sansoni, 1953. I racconti di questa raccolta - Storia d'amore (rielaborazione della precedente Storia di Debora e stesura che precede di qualche anno Lida Mantovani), La passeggiata prima di cena e Una lapide in Via Mazzini - erano usciti sulla rivista «Botteghe oscure» tra la fine degli anni'40 e l'inizio degli anni '50, rispettivamente nel 1948 sul I Quaderno, nel 1951 sul VII, nel 1952 sul X; inoltre alcuni racconti più brevi appaiono anche su quotidiani e periodici della stampa postbellica (è il caso, ad esempio, dei racconti che nel 1972 confluiranno nel volume L'odore del fieno: Il muretto di cinta, «Il Mondo», 6 aprile 1946, p. 7 e Meat and vegetables, «Il Popolo», 22 agosto 1946, p. 3). Lo scrittore perciò in questa fase sceglie due diverse strategie: per quanto riguarda la prosa, pubblica in modo sparso i suoi lavori in diverse sedi, mentre per quanto riguarda la poesia riunisce in volumi le liriche scritte negli anni precedenti.

19. G. Bassani, Storie dei poveri amanti e altri versi, Roma, Astrolabio, 1945; Te lucis ante, Roma, Ubaldini, 1947; Un'altra libertà, Milano, Mondadori, 1951.

20. La raccolta Un'altra libertà è suddivisa in due sezioni; la prima è appunto la ripresa di molte poesie della precedente Te lucis ante ed è dedicata a Dinda e Niccolò Gallo. La seconda sezione si intitola Dal Profondo ed è dedicata a Marguerite Caetani.

21. F. Fortini, Su questo momento di poesia, cit., p. 64.

22. «Le note precedenti sono state redatte [...] soprattutto tenendo presenti le riviste che pubblicano poesia contemporanea. Fra queste, i numeri 3, 4 e 5 di "Botteghe oscure", semestralmente edita a Roma [...]» (ibid.).

23. G. Bassani, Quattro poesie, «Botteghe oscure», nº V, II semestre 1950, pp.90-91. Con i titoli: Villa glori, Angelus, L'alba ai vetri, Sogno le poesie verranno raccolte in Id., L'alba ai vetri, Torino, Einaudi, 1963.

24. Cfr. P. Italia, Notizie sui testi, in G. Bassani, Opere, cit., p. 1780.

25. «Nella primavera del ' 42 , il primo impulso a scrivere versi mi venne, più che dalla vita e dalla realtà, dalla cultura. Da tempo mi avevano colpito le poesie di due vecchi compagni d'università, Francesco Arcangeli e Antonio Rinaldi [...]. Seguivo oltre a ciò i miei amici storici dell'arte, lo stesso Francesco Arcangeli, Giuseppe Raimondi, Carlo Ludovico Ragghianti, Cesare Gnudi, Giancarlo Cavalli, sulle tracce dei pittori ferraresi» (G. Bassani, Poscritto, in Id., Opere, cit., pp. 1162-1163).

26. Ivi, p. 1165.

27. F. Bartolini, Le prime raccolte poetiche di Bassani, in S. Amrani e M. P. De Paulis-Dalembert (a cura di), Bassani nel suo secolo, Ravenna, Giorgio Pozzi editore, 2017, p. 169.

28. Ivi, p. 167.

29. Ivi, p. 168.

30. J. Nimis, Lirismo dell'impegno nella prima stagione poetica, in Bassani nel suo secolo, cit., p. 183.

31. G. Bassani, Un'altra libertà, cit., p. 53. La poesia apparirà poi con il titolo Angelus nella raccolta L'alba ai vetri, Torino, Einaudi, 1963, p. 71. Con lo stesso titolo si trova all'interno di In rima e senza, in Opere, cit., p. 1398. 
32. G. Bassani, Poscritto, in Id., Opere, cit., p. 1165.

33. F. Fortini, Su questo momento di poesia, cit., p. 64.

34. G. Bassani, Poscritto, in Id., Opere, cit., p. 1165.

35. Cfr. F. Erbosi, Lettere tra Giorgio Bassani e Attilio Bertolucci (1937-1971), Tesi di laurea triennale svolta presso l'Università La Sapienza, sotto la guida della Prof.ssa P. Italia, a/a 2014/2015. È prevista per il 2018 la pubblicazione dell'edizione integrale del carteggio e il testo di una relazione sul rapporto tra Bassani e Bertolucci tenuta al convegno per il Centenario di Bassani nel novembre 2017 a Roma. Ringrazio Flavia Erbosi per avermi segnalato i due passi della corrispondenza tra Bassani e Bertolucci che saranno riportati.

36. F. Erbosi, Lettere tra Giorgio Bassani e Attilio Bertolucci (1937-1971), cit., p. 112. Le lettere di G. Bassani ad A. Bertolucci sono conservate presso l'Archivio di Stato di Parma.

37. Ivi, p. 111.

38. F. Fortini, Schedario, cit., p. 3.

39. Bassani venne giudicato da una giuria letteraria composta da Betocchi, Bo, De Robertis, Falqui, Flora, Piccioni e Vittorini che doveva scegliere, tra 345 opere concorrenti, oltre alla vincitrice, cinque da premiare con un premio in denaro. Bassani ottenne il terzo posto tra le opere segnalate e vinse 150000 lire. Si ricordano tra i presenti le più alte cariche dello Stato: il Presidente del consiglio De Gasperi, il Ministro dell'Istruzione Gonella, il Presidente del Senato Bonomi e il Presidente della Camera Gronchi. Per la cronaca della premiazione si rinvia all'articolo Lauree in Campidoglio. Ungaretti, Betti, Rossellini vincitori dei Premi Roma 1949, «La Fiera letteraria», 19 marzo 1950, p. 1.

40. P. Italia, Tra poesia e prosa: un percorso dal carteggio Bassani-Fortini (1949-1970), cit., p. 62.

41. G. Litrico, «Poor wounded soul!». Il carteggio tra Giorgio Bassani e Franco Fortini (1949-1970), Tesi di laurea magistrale svolta presso l'Università La Sapienza, sotto la guida della Prof.ssa P. Italia, a/a 2015/2016, p. 88.

42. Ivi, p. 168.

43. P. Italia, Tra poesia e prosa: un percorso dal carteggio Bassani-Fortini (1949-1970), cit., p. 62.

44. «[...] l'atteggiamento tipico di Bassani, luci fra lacrime, ha più ambiziose ascendenze, petrarchesche ("fanno le luci mie di piagner vaghe") raciniane (“J’ai langui, j’ai séché dans les yeux, dans les larmes") e leopardiane ("non brillin gli occhi tuoi se non di pianto")» (G. Litrico, «Poor wounded soul!». Il carteggio tra Giorgio Bassani e Franco Fortini (1949-1970), cit., p. 168).

45. Ivi, p. 93.

46. F. Diaco, Dialettica e speranza. Sulla poesia di Franco Fortini, Macerata, Quodlibet, 2017, p. 71.

47. G. Raboni, Intellettuale e poeta, «L'Immaginazione», $\mathrm{n}^{\circ}$ 130, giugno 1996, p. 14. Ora in F. Diaco, Dialettica e speranza. Sulla poesia di Franco Fortini, cit., p. 51.

48. L. Lenzini, Introduzione a Fortini saggista, cit., p. 25.

49. In particolare pubblica i testi su «La Riforma letteraria» di G. Noventa, come si può leggere nella Bibliografia delle opere fortiniane (Indici per Fortini. Con due contributi di Franco Fortini, a cura di C. Fini, L. Lenzini e P. Mondelli, Firenze, Le Monnier, 1989, p. 34).

50. F. Fortini, Solitudine di Ungaretti, Solitudine di Quasimodo, «La Riforma letteraria», $\mathrm{n}^{\circ} 28$, aprile 1939, pp. 6-11.

51. L. Daino, Un'interpretazione partigiana del passato, ACME-Annali della Facoltà di Lettere e Filosofia dell'Università degli studi di Milano, vol. LX, nº 1, gennaio-aprile 2007, p. 211.

52. Ibid.

53. Ivi, p. 212.

54. Ivi, p. 217.

55. B. De Luca (a cura di), Foglio di via e altri versi di Franco Fortini. Edizione critica e commentata, Tesi di dottorato svolta presso l'Università degli Studi di Napoli Federico II, a/a 2015/2016, p. 139.

56. L. Daino, Un'interpretazione partigiana del passato, cit., p. 216. 
57. B. De Luca (a cura di), Foglio di via e altri versi di Franco Fortini. Edizione critica e commentata, cit., p. 143.

58. G. Bassani, Storie dei poveri amati, e altri versi, Roma, Astrolabio, 1945, p. 5.

59. B. De Luca (a cura di), Foglio di via e altri versi di Franco Fortini. Edizione critica e commentata, cit., p. 176.

60. G. Bassani, Dopo la sagra, in Id., Storie dei poveri amanti e altri versi, cit., p. 16; la poesia apre la raccolta L'alba ai vetri, cit., p. 11, con titolo diverso, Dopo la sagra di San Giorgio.

61. Ivi, p. 17.

62. F. Bartolini, Le prime raccolte poetiche di Bassani, cit., p. 178.

63. Ivi, p. 176.

64. Ivi, p. 177.

65. G. Litrico, «Poor wounded soul!». Il carteggio tra Giorgio Bassani e Franco Fortini (1949-1970), cit., p. 169.

66. F. Fortini, Poesia di Bassani, cit., p. 76.

67. Ibid.

68. F. Fortini, Quel giovane tedesco, in Id., Tutte le poesie, a cura di L. Lenzini, Milano, Mondadori, 2014, p. 76.

69. G. Bassani, Poscritto, cit., p. 1165.

70. F. Fortini, Prefazione, in Foglio di via e altri versi, Torino, Einaudi, $1967^{2}$ (ed. origin. 1946), p. 8.

71. Fortini scrive questa frase nella lettera a Bassani del 4 gennaio 1951 (G. Litrico, «Poor wounded soul!». Il carteggio tra Giorgio Bassani e Franco Fortini (1949-1970), cit., p. 78).

72. G. Mazzoni, Sulla poesia moderna, Bologna, Il Mulino, 2015 (I ed. 2005), p. 204.

73. F. Fortini, Tutte le poesie, a cura di L. Lenzini, cit., p. XIV.

74. F. Diaco, Dialettica e speranza. Sulla poesia di Franco Fortini, cit., p. 132.

75. L. Lenzini, Traducendo Brecht, in Id., Il poeta di nome Fortini, Lecce, Manni, 1999, p. 129.

76. F. Diaco, Dialettica e speranza. Sulla poesia di Franco Fortini, cit., p. 137.

77. L. Lenzini, Traducendo Brecht, cit., p. 130.

78. G. Litrico, «Poor wounded soul!». Il carteggio tra Giorgio Bassani e Franco Fortini (1949-1970), cit., p. 68.

79. Ibid.

80. Ivi, p. 78.

81. L. Lenzini, Traducendo Brecht, cit., p. 131.

82. G. Bassani, L'alba ai vetri, in Id., Un'altra libertà, cit., p. 54.

83. G. Bassani, Perché dall'avvenire, ivi, p. 50.

84. Ibid.

85. G. Bassani, Come i primi ricordi, ivi, p. 62.

86. F. Fortini, Le poesie italiane di questi anni, in Saggi italiani, cit., poi in Saggi ed epigrammi, cit., pp. 548-606.

87. In Un'altra libertà Bassani solo in due circostanze (E non resti di me che un grido, p. 46 e Perché dall'avvenire, p. 50) usa parole della stessa radice (rispettivamente «tremito» e «tremante») ma mai «tremore».

88. Cfr. L. Lenzini, Traducendo Brecht, cit., p. 131 e F. Diaco, Dialettica e speranza. Sulla poesia di Franco Fortini, cit., p. 134.

89. F. Diaco, Dialettica e speranza. Sulla poesia di Franco Fortini, cit., p. 30.

90. I destini generali è il titolo di una poesia (F. Fortini, Tutte le poesie, cit., p. 177) e di una plaquette di versi fortiniani (F. Fortini, I Destini generali. Con uno scritto di poetica, Caltanissetta, Sciascia editore, 1956).

91. G. Litrico, «Poor wounded soul!». Il carteggio tra Giorgio Bassani e Franco Fortini (1949-1970), cit., p. 154. 


\section{RIASSUNTI}

Alla luce dei nuovi documenti emersi dall'Archivio epistolare di Giorgio Bassani, il saggio propone un esame della riflessione critica di Franco Fortini intorno alle opere dello scrittore ferrarese. L'obiettivo di questo studio è mettere a fuoco i primi anni del dialogo critico tra Bassani e Fortini, e in particolare il biennio 1950-1952, durante il quale lo sguardo di Fortini si sofferma sulle raccolte poetiche Te lucis ante (1947) e Un'altra libertà (1951).

Nella prima parte del saggio si è ricostruito il punto di vista di Bassani sugli scritti fortiniani relativi alla sua poesia e si è avanzata l'ipotesi che il testo di Bassani Poscritto (1952) sia stato ideato proprio in seguito alle discussioni avute con l'amico. Nella seconda parte si sono ricostruite le ragioni delle critiche mosse da Fortini a Bassani, ragioni legate alla sua esperienza poetica prebellica. Infine, si è tentato di verificare come, al di là di quelle critiche, la produzione poetica di Fortini in quegli anni porti a sua volta le tracce del dialogo con Bassani.

Cet essai, qui s'appuie sur les documents récemment repérés dans les archives Bassani à Paris (« Archivio Eredi Bassani ») propose une analyse des études critiques consacrées par Fortini aux deux premiers recueils poétiques de Bassani : Te lucis ante (1947) et Un'altra libertà (1951).

La première partie de l'étude se focalise sur la réaction de Bassani aux comptes rendus de Fortini et avance l'hypothèse que le Poscritto rédigé par Bassani en 1952, ait été conçu par l'auteur comme une réponse aux objections de son ami. La deuxième partie s'efforce d'expliquer l'attitude critique de Fortini vis-à-vis de Bassani par l'évolution de sa propre poétique ainsi que par sa volonté de se démarquer des poèmes que lui-même avait écrits avant la guerre. Il semble néanmoins que le débat entre les deux poètes a aussi laissé des traces dans la production poétique de Fortini.

This essay, based on documents from Bassani's archives in Paris ("Archivio Eredi Bassani"), proposes an analysis of Fortini's published reviews and private commentaries on Bassani's work. The essay focuses on the first years of the critical dialogue between the two poets, a period of a couple of years (1950-1952), during which Fortini examined the two early poetical collections of his friend: Te lucis ante (1947) and Un'altra libertà (1951).

The first part of the discussion concentrates on Bassani's response to Fortini's writings on his poetry and suggests that Bassani's 1952 Poscritto may have been written as a reaction to his friend's criticism and as an attempt to justify his own poetry. The second part examines the context within which Fortini criticizes Bassani's poetry. On the one hand, Fortini's objections to Bassani may be regarded as a way for him to distance himself from his own pre-war poetry. On the other hand, the evolution of Fortini's poetry in the following years suggests that Bassani may have had, after all, some positive impact on Fortini's conception and practice of poetry.

\section{INDICE}

Mots-clés : poésie du XXe siècle, archives littéraires, Te lucis ante, Un'altra libertà, Foglio di Via, Poscritto, littérature d'après-guerre, «Comunità», «Avanti!»

Parole chiave : poesia del Novecento, archivi d'autore, Te lucis ante, Un'altra libertà, Foglio di Via, Poscritto, letteratura del dopoguerra, «Comunità», «Avanti!»

Keywords : 20th century poetry, literary archives, Te lucis ante, Un'altra libertà, Foglio di Via, Poscritto, postwar literature, "Comunità", “Avanti!” 
AUTORE

GAIA LITRICO

Sapienza università di Roma 